\title{
Persée
}

http://www.persee.fr

\section{"Dépôts", "réserves" et "caches" de matériel lithique taillé au Néolithique précéramique au Proche-Orient : quelle gestion de l'outillage ?}

GONZÁLES URQUIJO J.;IBÁÑEZ ESTÉVEZ J. J.;ABBÈS F.;ASTRUC L.

\author{
Paléorient, Année 2003, Volume 29, Numéro 1
}

p. $59-78$

Voir l'article en ligne

\section{Avertissement}

L'éditeur du site «PERSEE »- le Ministère de la jeunesse, de l'éducation nationale et de la recherche, Direction de l'enseignement supérieur, Sous-direction des bibliothèques et de la documentation - détient la propriété intellectuelle et les droits d'exploitation. A ce titre il est titulaire des droits d'auteur et du droit sui generis du producteur de bases de données sur ce site conformément à la loi n`98-536 du $1 \mathrm{er} \mathrm{juillet} 1998$ relative aux bases de données.

Les oeuvres reproduites sur le site «PERSEE » sont protégées par les dispositions générales du Code de la propriété intellectuelle.

Droits et devoirs des utilisateurs

Pour un usage strictement privé, la simple reproduction du contenu de ce site est libre.

Pour un usage scientifique ou pédagogique, à des fins de recherches, d'enseignement ou de communication excluant toute exploitation commerciale, la reproduction et la communication au public du contenu de ce site sont autorisées, sous réserve que celles-ci servent d'illustration, ne soient pas substantielles et ne soient pas expressément limitées (plans ou photographies). La mention Le Ministère de la jeunesse, de l'éducation nationale et de la recherche, Direction de l'enseignement supérieur, Sous-direction des bibliothèques et de la documentation sur chaque reproduction tirée du site est obligatoire ainsi que le nom de la revue et- lorsqu'ils sont indiqués - le nom de l'auteur et la référence du document reproduit.

Toute autre reproduction ou communication au public, intégrale ou substantielle du contenu de ce site, par quelque procédé que ce soit, de l'éditeur original de l'oeuvre, de l'auteur et de ses ayants droit.

La reproduction et l'exploitation des photographies et des plans, y compris à des fins commerciales, doivent être autorisés par l'éditeur du site, Le Ministère de la jeunesse, de l'éducation nationale et de la recherche, Direction de l'enseignement supérieur, Sous-direction des bibliothèques et de la documentation (voir http://www.sup.adc.education.fr/bib/ ). La source et les crédits devront toujours être mentionnés. 


\title{
« DÉPÔTS », « RÉSERVES » ET « CACHES » DE MATÉRIEL LITHIQUE TAILLÉ AU NÉOLITHIQUE PRÉCÉRAMIQUE AU PROCHE-ORIENT : QUELLE GESTION DE L'OUTILLAGE?
}

\author{
L. ASTRUC, F. ABBÈS, J. J. IBÁÑEZ ESTÉVEZ et J. GONZÁLEZ URQUIJO
}

\begin{abstract}
Résumé : « Dépôts 》, "réserves » et " caches » de matériel lithique taillé sont mentionnés à plusieurs reprises pour le Néolithique précéramique au Proche-Orient. S'interroger sur ce que ces contextes particuliers, souvent divers par leur localisation, leur contenant et leur contenu, reflètent de l'organisation économique et sociale des communautés néolithiques est au centre de cet article. Le rôle technique et économique (gestion de l'outillage) de ce type de stockage est d'abord abordé. Parmi les facteurs qui président à la constitution de ces concentrations, nous examinons ensuite les facteurs sociologiques qui semblent dominants. Mécanismes de production et de redistribution de l'industrie lithique taillée au sein des communautés, degrés de spécialisation ou échelles de production sont alors évoqués dans une perspective diachronique.
\end{abstract}

\begin{abstract}
Caches and hoards of chipped stone artefacts are mentioned in the Near East during the Pre-Pottery Neolithic. These specific concentrations are often diverse in their location and their nature as both container and content. The question asked in the following pages is : how far are these particular contexts relevant as a way to characterize part of the economical and social organization of neolithic communities? The technical and economical role of this type of storage is first adressed. Among the factors that determine the formation of such clusters, we then argue that the sociological component is dominant. The mecanisms of production and redistribution of lithic tools within communities, the different levels of specialization or scales of production are at the end discussed in a diachronic perspective.
\end{abstract}

Mots Clefs : Cache, Stockage, Industrie lithique, PPN, Spécialisation, Proche-Orient, Chypre.

Key-Words : Cache, Storage, Lithic Industry, PPN, Specialization, Near East, Cyprus.

\section{INTRODUCTION}

« Dépôts », « réserves » et « caches » de matériel lithique taillé sont signalés sur les sites du Néolithique précéramique au Proche-Orient. Ces termes dont l'emploi n'est que rarement justifié désignent une concentration de matériel particu- lière, qui se distingue des contextes plus courants de zones d'activité ou de zones de rejet. L'identification sur le terrain de telles concentrations, souvent délicate - les contextes clos étant rares -, repose généralement sur trois critères : des objets sont découverts groupés; le contexte de trouvaille - localisation, contenu, contenant - indique que l'ensemble a été volontairement constitué (intentional deposit) ; le maté- 
riel est, enfin, considéré comme étant mis de côté, stocké pour une utilisation future, il serait donc potentiellement utile et/ou investi d'une valeur symbolique (purposeful deposit). Dans la littérature anglo-saxonne, les termes cache ou hoard, les plus fréquemment utilisés, désignent tous deux assez indifféremment des dépôts de matériel lithique réalisés dans un but précis ${ }^{1}$.

Définir dans quelles intentions ces ensembles ont été constitués est ardu : s'agit-il de dépôts permanents ou non? dans quelle mesure répondent-ils à des besoins utilitaires ou relèvent-ils de la sphère symbolique $?^{2}$ Les questions les plus fréquemment posées dans la littérature restent difficiles à aborder.

Nous avons choisi, dans cet article, d'analyser les " dépôts ", " réserves » et "caches » de matériel lithique taillé du Néolithique précéramique au Proche-Orient pour atteindre un objectif particulier. L'étude est, en effet, conduite afin de mieux définir les mécanismes de production et de distribution de l'outillage au sein des communautés et à l'extérieur de celles-ci. C'est en cela, plus qu'en évoquant le domaine symbolique, que nous souhaitons apporter des informations sur l'organisation économique et sociale des communautés étudiées.

La réflexion que nous proposons sera conduite en trois parties. Dans la première, nous dressons l'inventaire des données dont nous disposons, selon un système descriptif homogène - localisation, contenant et contenu - en employant les termes neutres de concentration ou d'ensemble. Cet inventaire nous conduit à constater une diversité importante des contextes.

La deuxième partie est consacrée à une discussion générale sur l'interprétation de ces contextes. Nous proposons d'abord de définir les termes "dépôt", "réserve" et " cache » selon des critères précis. Ces critères seront ensuite examinés en abordant la question du mode de constitution et de fonctionnement de ces ensembles. Les questions suivantes seront notamment traitées : quels sont les facteurs qui président à leur constitution? dans quelle mesure les "dépôts ",

1. J. Levy proposait pour le terme hoard une définition large : « objects deposited purposefully » (LEVY, 1982 : 13), définition qui peut alors regrouper des expressions diverses plus ou moins interprétatives: caches, foundation deposits, mortuary deposits, bothroi, votive deposits, dedicatory offerings, etc. D'autres auteurs réservent le terme hoard à des comportements relevant plus de la sphère rituelle que du domaine utilitaire ( $c f$. PELTENBURG, 1991).

2. En ce qui concerne les discussions sur la valeur symbolique des productions lithiques et des concentrations de matériel lithique, $c f$. notamment, CAUVIN, 1994 : 169 ; BAR-YOSEF and ALON, 1988 ; GORING-MORRIS and BELFER-COHEN, 2001 ; CONOLLY, 2003. les « réserves » et les " caches » de matériel lithique répondent-ils à une recherche d'une gestion optimale des ressources lithiques? quel est leur impact économique? peuvent-ils témoigner des mécanismes de production et de redistribution de l'outillage au sein d'une communauté ?

La troisième partie place ces interrogations dans une perspective diachronique. Il nous est alors possible de montrer dans quelle mesure les "dépôts ", " réserves " et " caches " de matériel lithique sont le reflet des différents contextes socioculturels du Néolithique précéramique au ProcheOrient.

\section{UNE DIVERSITÉ DE CONTEXTES}

Les données dont nous disposons pour le Proche-Orient (fig. 1) vont être exposées en suivant un axe chronologique, du PPNA jusqu'à la fin du Néolithique précéramique. Cet inventaire nous permet de constater la variété des situations archéologiques rencontrées et de mettre en évidence une évolution des comportements de stockage de matériel lithique au cours du Néolithique précéramique.

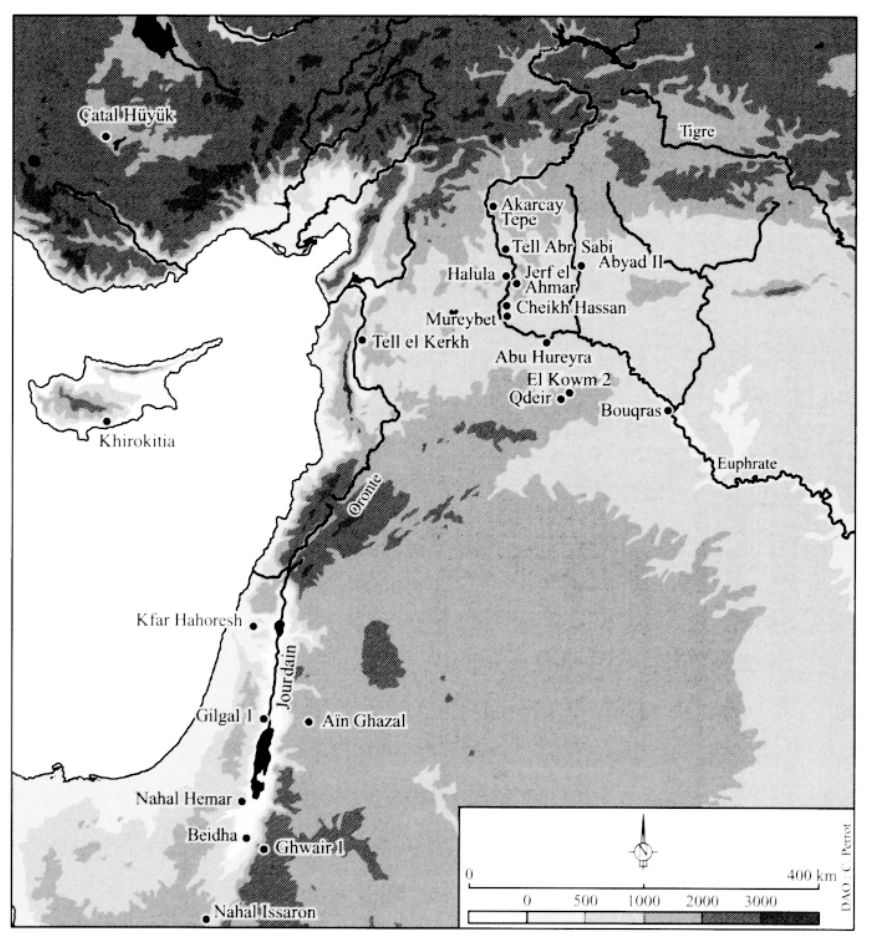

Fig. 1 : Carte de localisation des sites cités dans le texte. 
Les contextes de trouvaille sont extrêmement variés. Les concentrations se trouvent dans les habitats fouillés directement associées aux constructions ou dans les espaces nonbâtis attenants. À l'intérieur des maisons, des éléments lithiques peuvent être regroupés dans divers contenants, un panier suspendu (Gilgal 1, début du $\mathrm{PPNA}^{3}$ ), une boîte en bois (Beidha, niveau VI, PPNB ${ }^{4}$ ) ou encore, une fosse creusée dans le sol d'une maison ('Ain Ghazal, PPNB, Jordanie ${ }^{5}$ ), une cuvette (Mureybet IIIA, PPNA ${ }^{6}$ ), une niche (Jerf el Ahmar, transition $\mathrm{PPNA} / \mathrm{PPNB}^{7}$ ) ou une structure domestique (Ghwair8 I, début du PPNB moyen). Dans d'autres cas, des concentrations de matériel sont trouvées dans une pièce ou un réduit, en vrac ou en fagot, et parfois associées à d'autres catégories de matériel (Abu Hureyra ${ }^{9}$ et Sabi Abyad II ${ }^{10}$, PPNB récent; Khirokitia, Néolithique précéramique récent ${ }^{11}$ ) ou encore, plus exceptionnellement, dans un coin de la cour d'une maison (Bouqras, PPNB final ${ }^{12}$ ) ou dans une fosse creusée dans le sol d'un espace non-couvert (Jerf el Ahmar, transition PPNA/PPNB, à Cheikh Hassan ${ }^{13}$ au PPNB ancien, à Akarçay Tepe ${ }^{14}$ au PPNB récent). Enfin, des concentrations de lames sont trouvées déconnectées de tout contexte architectural (el-Kerkh, PPNB récent ${ }^{15}$ ).

À cette diversité des contextes de trouvaille, s'ajoute la variété des contenus puisque le matériel stocké peut comprendre, selon les cas, des nucléus, des supports et/ou des outils.

\section{AU PPNA}

Pour le PPNA, nous disposons de deux exemples l'un, à Gilgal 1 et l'autre, à Mureybet IIIA. À Gilgal 1, tout le matériel lithique de la maison 11 a été trouvé groupé sur le sol, associé à une empreinte de panier enduit de bitume ${ }^{16}$. La disposition des éléments a conduit le fouilleur à interpréter l'ensemble comme le résultat de la chute d'un panier suspendu contenant un maté-

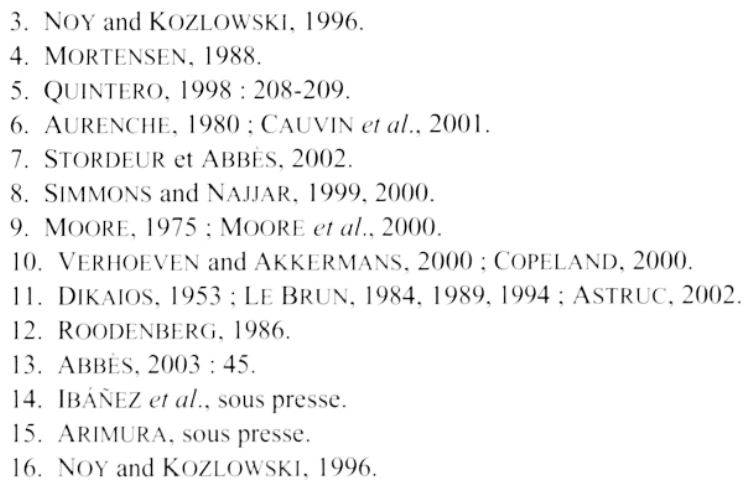

riel lithique divers et abondant ( 147 pièces): des éclats et débris ${ }^{17}$ (84), des lames et éclats bruts (7), des lames et lamelles avec retouche d'utilisation (39) et des outils retouchés (13, lames retouchées, faucilles, troncatures, perçoirs, burin et pic), mais aussi des nucléus (4). La comparaison de cet ensemble avec le reste de l'industrie lithique des niveaux 1 et 2 correspondants a montré : une forte proportion de pièces techniques laminaires, d'éclats et de pièces retouchées ; l'absence de pointes de flèche et de grattoirs; la sélection d'éclats dont beaucoup sont corticaux, de lames entières et fractionnées à part égale et de lamelles entières. Les auteurs proposent alors pour cet ensemble le concept de «living inventory » (réserve) qui s'oppose à celui de "refuse inventory" (poubelle ou rejet). La concentration représenterait, en effet, non pas un rejet, mais un stock de matières premières et d'outils utilisés ou non.

À Mureybet IIIA ${ }^{18}$, une "cache " a été signalée par les fouilleurs dans la maison XLVII ${ }^{19}$, une construction ronde, enterrée dont l'espace interne est divisé par des murs de cloisonnement. Le matériel trouvé sur le sol de cette structure incendiée est en place. Dans l'une des cellules (A), au pied du muret 17 , une cuvette contenait 80 outils bruts ou retouchés (notamment, des grattoirs, burins, outils perçants, pointes de flèche et lames retouchées). Il s'agit du seul exemple dont nous disposons de concentration formée à la fois d'outils en obsidienne ( 1 racloir, 1 fragment de flèche et 1 lame) et d'éléments divers en silex. L'analyse fonctionnelle de douze lames retouchées et onze grattoirs a donné des résultats classiques pour cette période : les grattoirs sont associés au raclage de la peau, les pointes montrent des traces d'impact, les lames retouchées ont été utilisées dans le cadre de plusieurs activités (coupe et raclage de bois, raclage de plantes siliceuses, etc.). L'ensemble analysé montre aussi bien des outils neufs que des outils faiblement utilisés, ou déjà réaffûtés ou recyclés ${ }^{20}$.

\section{AU MOMENT DE TRANSITION ENTRE PPNA ET PPNB}

Les concentrations identifiées à Jerf el Ahmar se placent dans la phase de transition entre le PPNA et le PPNB (autour

\footnotetext{
17. La présence de débris indiquerait plutôt qu'il s'agit d'un matériel provenant à la fois du sol et du panier.

18. CAUVIN et al, 2001

19. AURENCHE, 1980.

20. D'autres concentrations d'outils particuliers ont été découvertes dans cette maison : de l'outillage lourd, dans les cellules $\mathrm{G}$ et $\mathrm{H}$, et de l'industrie osseuse, dans la cellule A. Le matériel repoussé le long des murs, souvent divers - une situation on ne peut plus fréquente dans les habitats néolithiques sort du cadre de cette étude.
} 
de $8700 \mathrm{cal}$. BC) ${ }^{21}$. C'est à ce moment qu'apparaissent un nouveau type de bâtiment à caractère communautaire et les premiers débitages de lames prédéterminées ${ }^{22}$ sur nucléus bipolaires, annonciateurs des technologies du PPNB.

La première concentration a été trouvée dans une petite fosse creusée dans un sol extérieur correspondant à un groupe de maisons de plan rectangulaire; ce sol est postérieur à la phase d'abandon d'un bâtiment communautaire (bâtiment EA53) (fig. 2). Elle est constituée de 15 lames (12 entières et 3 fragments) issues de 4 nucléus bipolaires différents ; certaines remontent entre elles. Ces supports de profil rectiligne mais de morphologie irrégulière s'insèrent dans la variabilité laminaire commune des débitages bipolaires, mais ne sont pas des lames prédéterminées ; ils correspondent aux lames utilisées, non pas pour la fabrication de pointes de projectile, mais pour celle d'une gamme importante d'outils (couteaux, burins, perçoirs...).

La deuxième concentration a été trouvée dans un contexte similaire - une fosse creusée dans un sol extérieur, postérieur à l'abandon d'un autre bâtiment communautaire (bâtiment EA30) (fig. 3) -, mais l'emplacement de la fosse était cette fois signalé en surface par un petit amoncellement de quatre pierres. La concentration comporte 4 nucléus bipolaires à lames ( 2 nucléus à crête postéro-latérale, 1 naviforme, 1 nucléus épuisé ne portant plus les traces d'une mise en forme probablement réalisée par une crête frontale ${ }^{23}$ ), des lames débordantes (dont certaines remontent sur les nucléus) et des éclats (dont une ouverture de plan de frappe). Les nucléus ont très visiblement produit des lames prédéterminées ; ils sont épuisés mais n'ont pas été abîmés par des enlèvements postérieurs au plein débitage. Le silex en rognon, de très bonne qualité, provient de la formation de Maskar ${ }^{24}$, une matière abondante à proximité du site, mais paradoxalement peu utilisée sur le site. La maîtrise et le savoir-faire dont les nucléus de cette fosse témoignent n'ont que peu d'équivalents dans l'habitat.

Une troisième concentration était située à l'intérieur d'une niche aménagée dans le mur d'une habitation de plan rectangulaire (maison EA5425 ; fig. 4). La niche était scellée et son con-

21. STORDEUR et ABBĖs, 2002

22. L'expression "lame prédéterminée" a été préférée par l'un des auteurs à celle de « lame préférentielle ", ceci notamment pour caractériser ces supports à Jerf el Ahmar et à Cheikh Hasssan (ABBĖS, $2003: 27-30$ ).

23. ABBÉS, $2003: 27$, pl. 8 .

24. L'affleurement connu le plus proche est situé à une trentaine de kilomètres du site (prospections F. Abbès). La localisation des sources est cependant rendue difficile par la construction de barrages sur l'Euphrate et par la montée des eaux du fleuve.

25. STORDEUR et ABBĖS, 2002 : fig. 10, 579. tenu, dissimulé. Six lames bipolaires prédéterminées y étaient déposées. Elles témoignent d'une qualité de débitage comparable à celle des nucléus précédemment cités ; les lames prédéterminées retrouvées dans ces niveaux de la phase de transition sont presque toutes retouchées et de moins bonne facture.

Enfin, il semblerait que des lames brutes et retouchées (couteaux et pointes de flèche), parfois prédéterminées, aient été déposées dans les enduits muraux intérieurs d'un bâtiment communautaire (bâtiment EA53) ; chaque support était pris dans l'enduit, la face plane placée contre le mur. Ce type d'observation doit être considéré avec prudence mais, dans ce cas précis, la fréquence de cette pratique et la nature des supports distincte des éléments de plus faibles modules contenus dans la terre à bâtir indiqueraient qu'il ne s'agit pas de pollutions.

Ainsi, nous nous trouvons, à Jerf el Ahmar, dans une même phase chronologique, la phase de transition PPNA/ PPNB, devant des situations que la localisation des concentrations différencie : des fosses creusées dans des sols extérieurs postérieurs à l'abandon des bâtiments communautaires, une niche dans le mur d'une habitation commune. À la diversité des contenants, s'ajoute sur ce site la variété des contenus; ces derniers montrent pourtant chacun une homogénéité interne et une spécificité, le résultat d'une ponction distincte au sein de chaînes opératoires différentes : ici, un lot de lames bipolaires communes extraites d'un même nucléus ou encore, un lot de nucléus épuisés associé à quelques pièces techniques leur correspondant, et là, un lot de lames prédéterminées.

Aucun de ces contextes ne présente de grandes concentrations d'objets. Les nucléus sont épuisés et ne peuvent donner lieu qu'à une production limitée d'éclats. La concentration numériquement la plus importante est constituée de quinze lames qui ne se distinguent pas de celles que l'on trouve par ailleurs en quantité sur le site. Cet ensemble et celui des six lames prédéterminées, s'ils doivent être interprétés comme des stocks de matériel, ne peuvent avoir qu'un faible impact économique. Nous verrons que ce constat peut être établi pour l'essentiel des contextes dont nous disposons (partie 2).

\section{AU PPNB ANCIEN}

À Cheikh Hassan ${ }^{26}$, une concentration de huit pointes, dont un fragment, a été découverte dans une petite fosse creusée dans le sol extérieur d'une habitation de plan rectangulaire, plu-

26. ABBÈS, 2003: 144, 146. Les niveaux sont contemporains de la phase IVA de Mureybet et du site de Dja'de (COQUEugNiOT, 2000). 

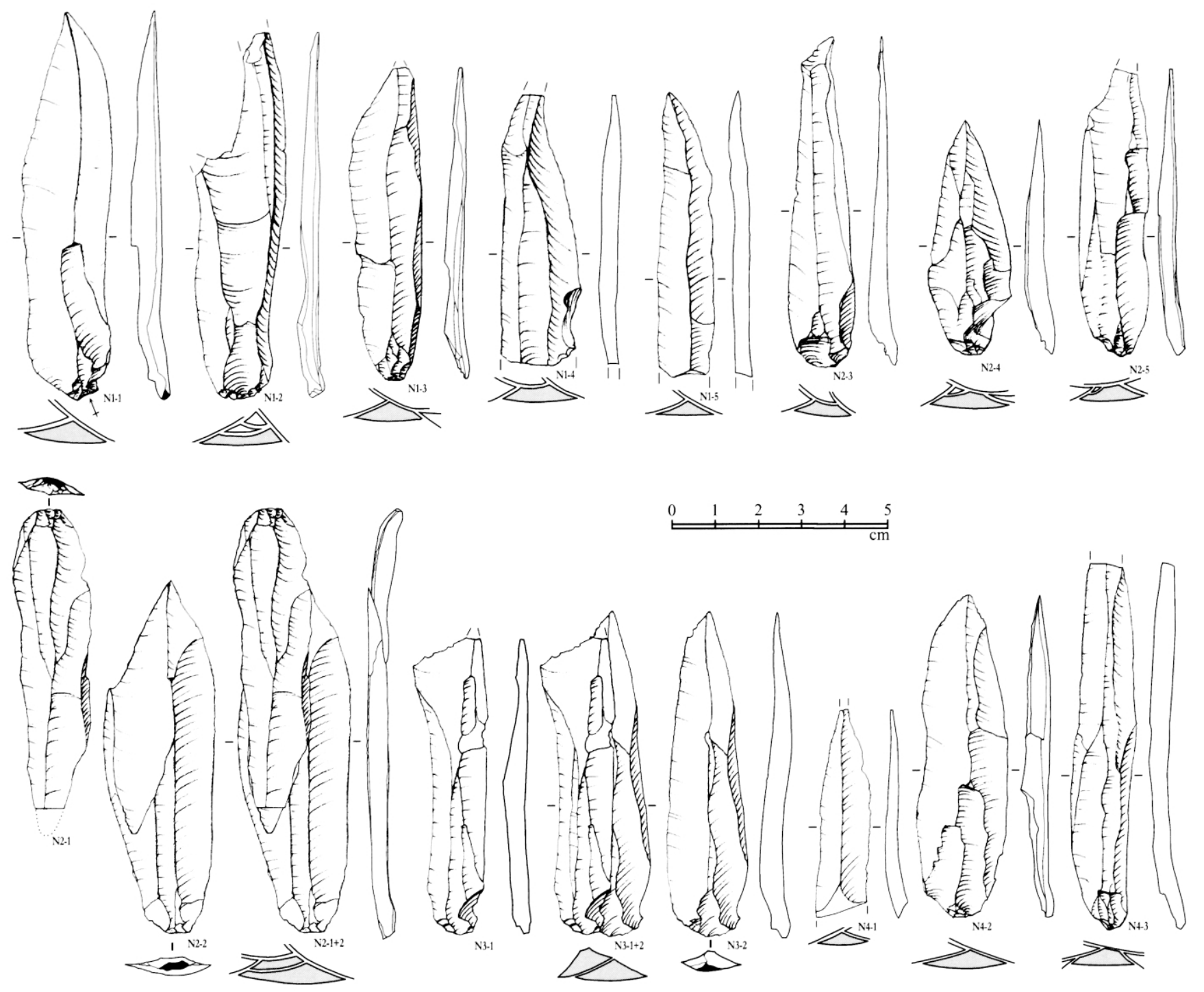

Fig. 2 : Jerf el Ahmar (phase de transition PPNA/PPNB).

Contexte 1. Quinze lames issues d'au moins quatre nucléus bipolaires différents : $1^{\text {er }}$ nucléus (N1-1/5), $2^{\mathrm{e}}$ nucléus (N2-1/5; avec remontage), $3^{\mathrm{e}}$ nucléus ( N3-1/2 ; avec remontage), $4^{\mathrm{e}}$ nucléus (N4-1/3).

ricellulaire (secteur FK). La composition de la concentration illustre plusieurs types de débitages laminaires (fig. 5): trois pointes proviennent de débitages bipolaires et l'une d'entre elles au moins est façonnée sur une lame prédéterminée ; cinq autres sont issues de débitages unipolaires dont quatre sont prédéterminées. Des silex d'origine fluviatile sont utilisés pour les débitages unipolaires et le silex de la formation de Maskar, sous forme de rognons, pour les débitages bipolaires.

Rien ne distingue vraiment le matériel de la concentration du reste de l'industrie lithique. Seule la très belle pointe sur lame prédéterminée est d'un type particulièrement rare sur le site; elle a pourtant pu être produite à proximité de l'espace domestique. Le matériel archéologique était peu abondant à l'intérieur de la construction et des restes osseux, un foyer et une meule en place trouvés immédiatement à l'extérieur de celle-ci témoignaient d'activités domestiques réalisées à immédiate proximité du bâtiment. Mais, c'est un peu plus loin que deux zones de taille ont été mises en évidence. La première est une aire de débitage domestique, de fabrication et d'utilisation d'outils qui témoigne d'une production d'éclats 

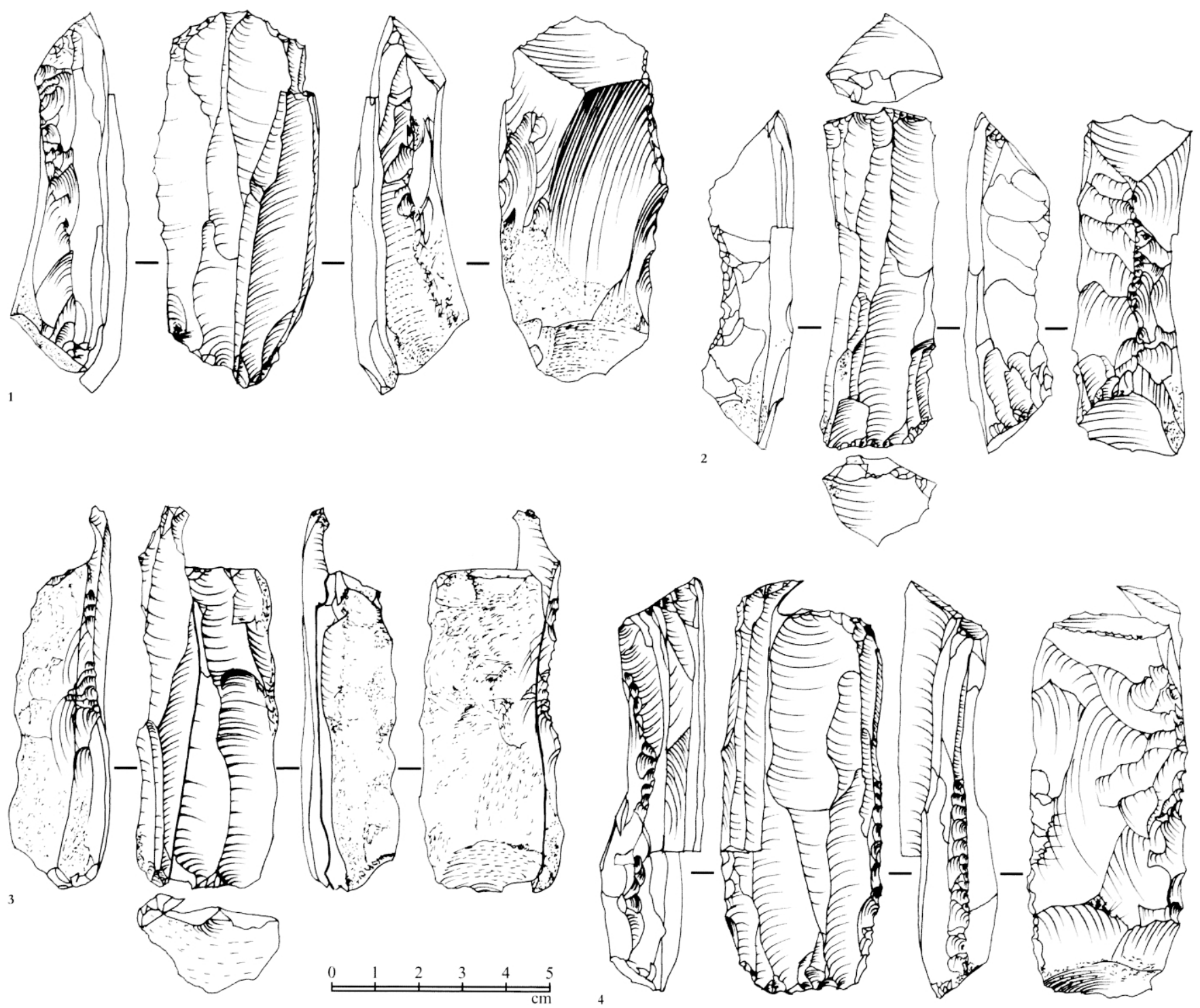

Fig. 3 : Jerf el Ahmar (phase de transition PPNA/PPNB).

Contexte 2. Quatre nucléus épuisés dont trois avec supports remontés : nucléus à crête postéro-latérale (1 et 4), nucléus naviforme (2), nucléus bipolaire (3).

et de l'exploitation terminale de nucléus à lames ( 17 nucléus, 373 lames et 1019 éclats). La seconde, un peu plus éloignée, est un atelier dévolu à la production de lames bipolaires prédéterminées. Or, l'analyse conjointe de ces zones a montré que des nucléus bipolaires, notamment naviformes, ont été exploités dans l'atelier avant d'être apportés dans la maison ou à proximité immédiate de celle-ci27.

27. ABBĖS, $2003: 75$.

\section{AU PPNB MOYEN}

À Beidha (niveau VI ${ }^{28}$ ), les fouilleurs ont découvert sur le sol de la maison incendiée XVIII, ce qui est interprété comme les vestiges d'une boîte en bois. Celle-ci contenait 114 pièces probablement enveloppées dans un cuir ou un tissu: 70 lames bipolaires non retouchées dont 3 lames à crête,

28. KIRKBrIDE, 1967 ; MORTENSEN, 1988. 

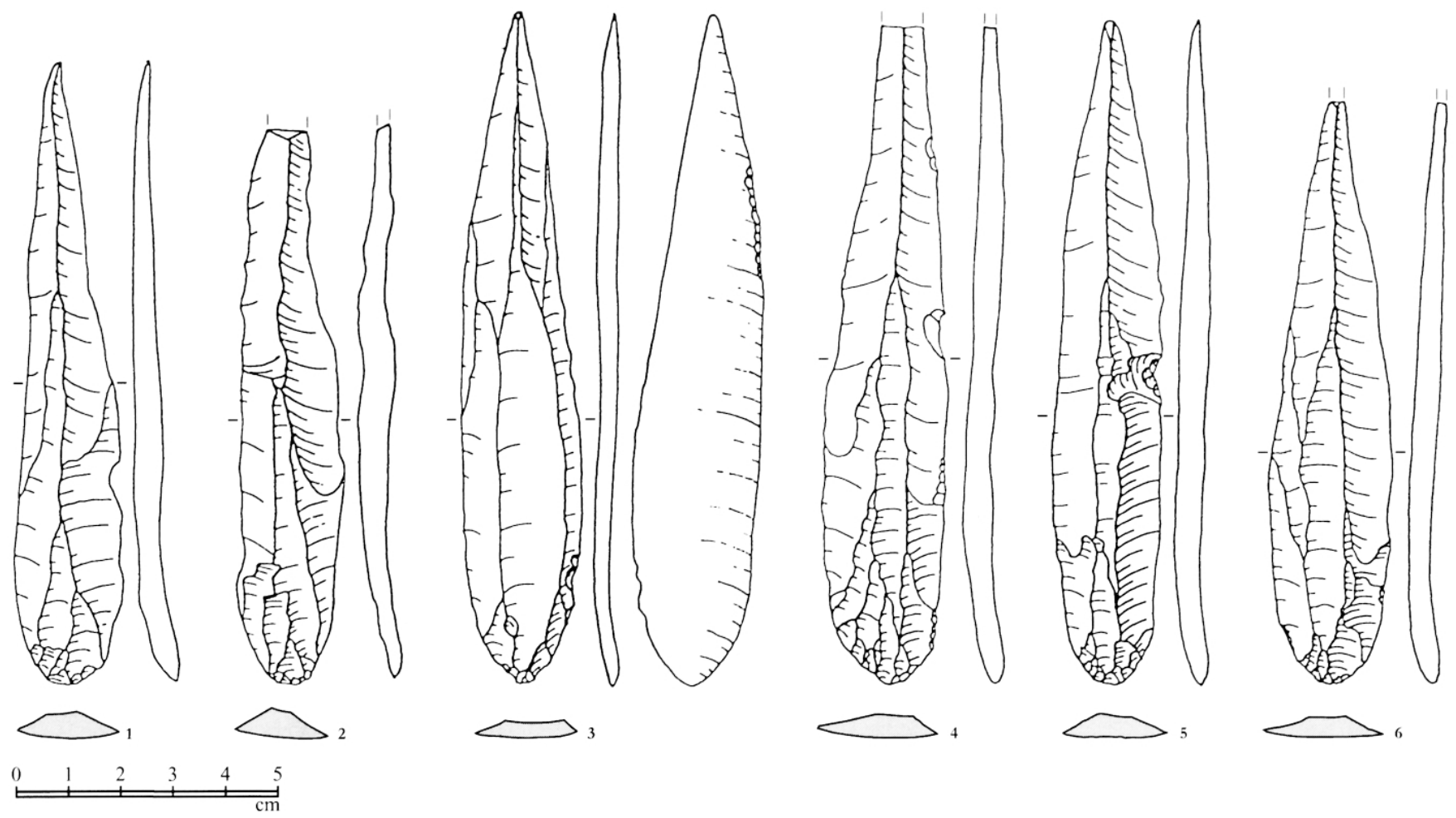

Fig. 4 : Jerf el Ahmar (phase de transition PPNA/PPNB).

Contexte 3. Lames bipolaires prédéterminées.

30 lames légèrement retouchées et 1 fragment distal, et 11 pointes sont associées à 2 nucléus fragmentés peut-être réutilisés comme retouchoirs ${ }^{29}$. L'auteur mentionne la présence de matières premières d'aspect varié (du silex tabulaire légèrement translucide, gris clair à brun-rouge) et de 8 lames en silex chocolat.

À 'Ain Ghazal $^{30}$, un groupe de 80 lames brutes en silex d'Huweijir a été trouvé sous le sol d'une maison. D'autres concentrations découvertes sous les sols des maisons ou dans leurs murs ont été brièvement mentionnées ${ }^{31}$.

À Ghwair $\mathrm{I}^{32}$, trois concentrations ( floor caches») de lames en silex ont été découvertes. 23 pointes de flèches (des pointes de Byblos et une pointe de Jéricho) ont été trouvées sur le sol d'une structure domestique («bin»; Area I). Deux

29. Cet ensemble est parfois décrit légèrement différemment : deux éclats et un fragment de nucléus auraient été trouvés associés aux 101 lames et 11 pointes (GORING-MORRIS and BELFER-COHEN, $2001: 260$ ).

30. QUINTERO, 1998: 208 ; trois variétés de silex rose et mauve.

31. BANNING and BYRD, 1987: 313 ; GORING-MORRIS and BELFERCOHEN, $2001: 263$.

32. SIMMONS and NAJJAR, 1999, 2000 autres ensembles présentaient des lames associées à des polissoirs et des fragments de malachite travaillée.

Des concentrations ont également été signalées sur les sites de Nahal Issaron, de Wabi Jibba ${ }^{33}$, de Kfar HaHoresh ${ }^{34}$ et de Halula ${ }^{35}$. Pour ce dernier site, un petit lot de trois lames préférentielles en silex chocolat ${ }^{36}$ a notamment été recueilli sur le sol du porche d'une maison. Ces pièces ne sont que peu ou pas utilisées; deux d'entre elles portent des traces de raclage de végétaux.

Il nous semble enfin que le contexte tout particulier de la grotte de Nahal Hemar ${ }^{37}$ considérée par certains comme une " cache » sort du cadre précis de cet article. Cette grotte a été interprétée comme un lieu de stockage de matériels divers et par-

33. GORING-MORRIS and BELFER-COHEN, $2001: 260$.

34. Il s'agit sur ce site de productions naviformes (N. Goring-Morris, comm. pers.).

35. MOLIST et al., 2001. L'analyse de la distribution spatiale des outils est en préparation.

36. Les indications concernant la nature des matières premières ont été fournies par J.J. Ibáñez Estévez.

37. BAR-YOSEF and ALON. 1988. 

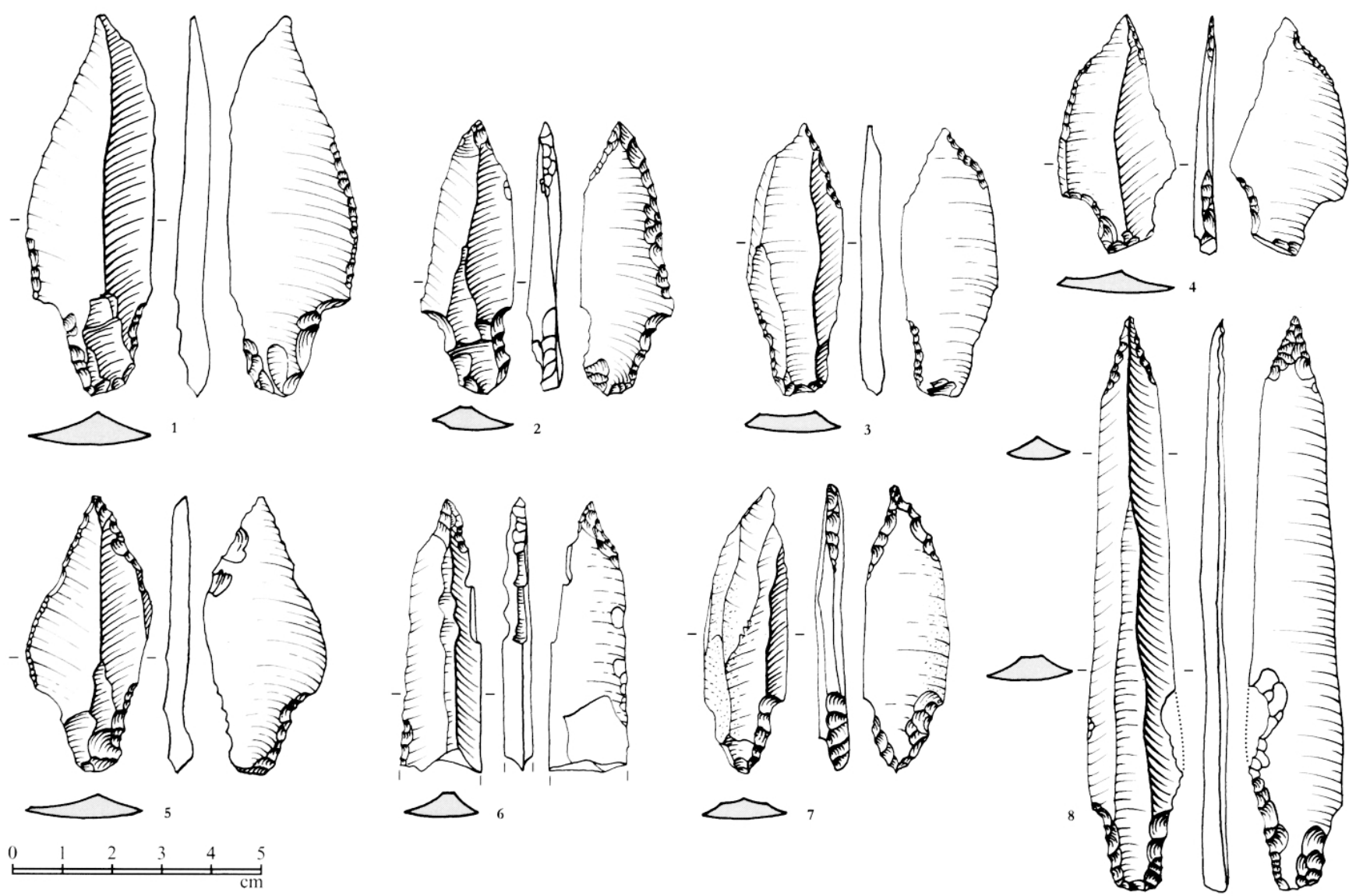

Fig. 5 : Cheikh Hassan (PPNB ancien).

Huit pointes : 1-5, unipolaires (1, 2, 4 et 5 sont prédéterminées) ; 6-8, bipolaires ( 8 est prédéterminée)

ticulièrement d'objets ayant une signification rituelle sans qu'il ne soit possible de déterminer en combien d'épisodes et selon quelles modalités cet ensemble s'est formé. Plus de 550 lames issues de débitages naviformes ont été trouvées là, associées à des crânes surmodelés, des masques en pierre, des statues anthropomorphes, des figurines et à divers matériaux organiques : des lames brutes ou partiellement retouchées, des perçoirs, des pointes de flèche, des éléments de faucille, des lames pointues, des pointes cassées et des couteaux de Nahal Hemar.

\section{AU PPNB RÉCENT ET FINAL}

À Abu Hureyra ${ }^{38}$, au sein d'un mobilier lithique abondant, une concentration de 230 lames en silex a été signalée. Ces

38. MOORE et al., 2000 : 244-248, fig. 8.65, 8.68, 8.69. lames brutes ou légèrement retouchées étaient groupées, probablement liées entre elles, dans une maison de la phase $2 \mathrm{~B}$. Plusieurs concentrations sont mentionnées sur ce site au PPNB récent ${ }^{39}$ et notamment, un petit ensemble de lames associées à un nucléus bipolaire ${ }^{40}$.

À Tell el-Kerkh ${ }^{41}$, un ensemble de 83 lames unipolaires a été recueilli dans un espace apparemment déconnecté de tout contexte architectural (Square D6). Les lames sont brutes, entières (40) ou fragmentées (43). Ces supports sont débités dans un silex local provenant de la formation de Zawiyeh, située à $1,5 \mathrm{~km}$ du site ${ }^{42}$. Quelques remontages ont été effectués; les lames seraient issues d'au moins trois nucléus

39. MOORE, $1975: 65$.

40. MOORE et al., 2000: 203.

41. ARIMURA, sous presse.

42. Cf. en ce qui concerne les matières premières, ARIMURA, 2003:57. 



Fig. 6 : Sabi Abyad II (PPNB récent).

Lames en obsidienne, débitées par pression.

débités par percussion indirecte ou par pression. Une hypothèse sur leur destination fonctionnelle a été proposée : leurs caractéristiques techno-morphologiques montrent qu'elles pouvaient être destinées à la fabrication d'éléments de faucille.

À Akarçay Tepe ${ }^{43}, 4$ nucléus bipolaires ont été trouvés groupés dans une fosse creusée dans un sol extérieur, à proximité d'une maison.

À l'exception du cas de Mureybet IIIA qui comporte quelques pièces en obsidienne, les exemples mentionnés jusque-là sont composés d'éléments en silex. Les deux seules concentrations d'obsidienne dont nous disposons datent du PPNB récent/final ${ }^{44}$. Elles ont été recueillies en Syrie, à Sabi Abyad II ${ }^{45}$ et à Bouqras ${ }^{46}$.

À Sabi Abyad $\mathrm{II}^{47}$, un petit ensemble d'une vingtaine de lames et fragments a été découvert dans une maison; aucune fosse n'a été mise en évidence et il semblerait que l'ensemble

43. ARIMURA et al., 2000.

44. Les concentrations d'obsidienne sont fréquentes à Catalhöyük, en Anatolie centrale, mais sortent du cadre de cet article (CONOLLY, 1999, 2003).

45. VERHOEVEN and AKKERMANS, 2000.

46. ROODENBERG, 1986.

47. Verhoeven and AKKermans, 2000: 10, fig. 2.5 ; COPELAND, 2000. ait été disposé en fagot ou dans un sac. Les lames ont toutes été débitées par pression ${ }^{48}$ (fig. 6). À Bouqras, un ensemble de trois cents lamelles en obsidienne a été trouvé dans un angle de la cour de la maison brûlée $n^{\circ} 12$ (espace 2$)^{49}$. Probablement liées en fagot, elles étaient complètes et fraîches tandis que les pièces entières ne constituent qu' $1 \%$ des lamelles recueillies par ailleurs dans l'habitat.

Sur chacun de ces deux sites, aucun témoin de débitage sur place d'obsidienne n'a été mis en évidence. Ce matériau qui provient de zones géographiques situées à plus de $300 \mathrm{~km}$ de là est importé sous forme de produits finis bruts, des lame(lle)s.

\section{AU NÉOLITHIQUE PRÉCÉRAMIQUE RÉCENT À CHYPRE}

À Khirokitia, différents types de concentrations de matériel lithique ont été caractérisés grâce à une analyse technolo-

48. L'étude techno-fonctionnelle en cours apportera de nouvelles informations sur le mode de constitution de cet ensemble.

49. ROODENBERG, $1986: 165$, note 11,179 , note 80 et 185 . 

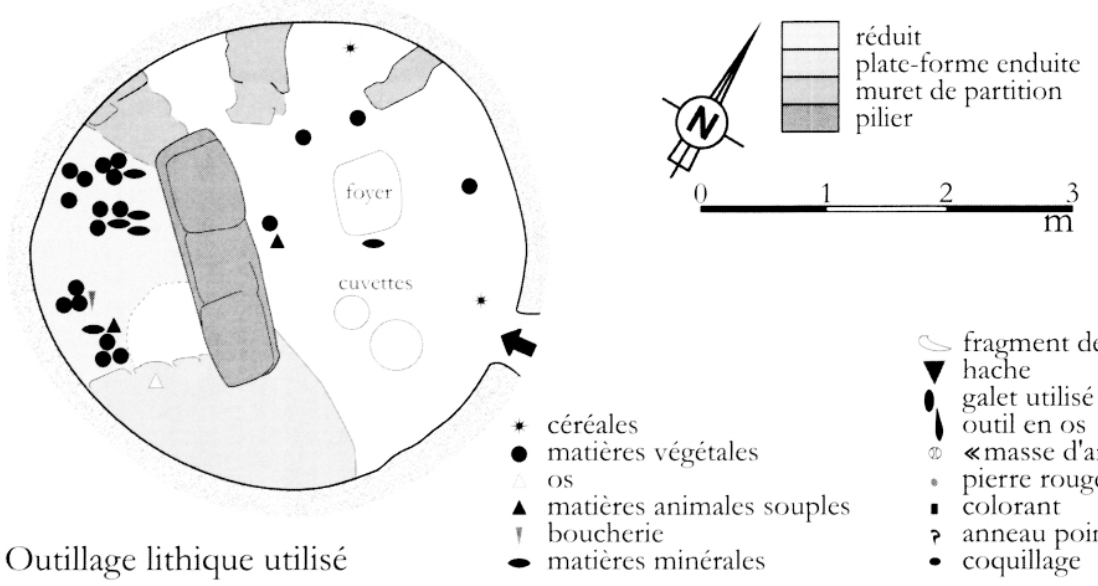

Outillage lithique utilisé

Fig. 7 : Khirokitia (Néolithique précéramique récent).

Construction S. 122, niveau C1. Localisation de la réserve de mobilier derrière le pilier. Sont figurés d'une part, les outils lithiques taillés utilisés (matières travaillées ou activité) et d'autre part, les objets divers associés.

gique et fonctionnelle, croisée avec une analyse de la distribution spatiale de l'ensemble du mobilier. Ont été distingués : d'une part, une concentration identifiée dans un réduit situé dans une des constructions circulaires du niveau $\mathrm{C}$ (S. $\left.122^{50}\right)$, réduit interprété comme un lieu de réserve de mobilier divers; et d'autre part, des concentrations d'outils qui correspondent vraisemblablement à des rejets liés à une activité technique précise.

La construction S. 122 est divisée en deux zones distinctes : une zone d'habitation proprement dite et un réduit, limité par un pilier, une plate-forme et un muret (fig. 7). Cet espace exigu mesure $3,48 \mathrm{~m}^{2}$ et est couvert d'un plancher situé à moins d'un mètre de hauteur, qui repose sur le pilier. Il ne s'agit donc pas d'un espace où l'on peut vivre ou travailler. Le matériel y a été trouvé à plat, disposé en lits successifs, ce qui montre une utilisation similaire de cet espace pendant un certain temps. L'industrie lithique taillée constitue l'essentiel du matériel du réduit mais elle est associée à d'autres catégories de matériel (fragments de vaisselle, galets utilisés, molette, "masse d'arme ", colorants et galets rouges, coquillage, anneau pointé en coquillage, outil en os). Ce mobilier divers n'est pas très différent de celui que l'on trouve dans la partie principale de l'habitation (fig. 7).

L'industrie lithique taillée comprend pour chacune des deux phases distinguées par les fouilleurs, respectivement 124

50. Pour l'analyse qualitative et quantitative détaillée de cet ensemble et la comparaison des données avec le reste de l'industrie, $c f$. ASTRUC, 2002. et 163 pièces en chert (formation locale de Lefkara ${ }^{51}$ ); de ces ensembles, il faut retrancher les débris, catégorie de matériel extrêmement répandue à Khirokitia puisqu'elle correspond, quels que soient les contextes, à près de $50 \%$ du lithique ${ }^{52}$. L'essentiel du matériel du réduit est constitué de supports très majoritaires (70 et 118), associés à quelques nucléus (3 et 6). Les supports retouchés sont assez rares ( 8 et 20) et les outils utilisés sont aussi épars ( 8 et 20). Aucun remontage n'a été identifié. Nous nous trouverions alors devant une situation archéologique proche du concept de "living inventory" (réserve) auquel nous avons déjà fait référence. Le mobilier est hétérogène et correspond à la fois à un stock de matière première (nucléus) et d'outils utilisés ou non mais réutilisables.

La composition de cet ensemble ne diffère pas, à de rares exceptions près du reste de l'industrie ${ }^{53}$ : des productions majoritairement laminaires, peu standardisées, vite produites, utilisées brièvement, ne faisant pas l'objet de ravivage ou de recyclage et donc vite rejetées (fig. $8: 1-4$ ). D'un point de vue fonctionnel, la gamme d'activités reconnues parmi les outils du réduit témoigne, comme ceux du reste de l'habitation, de petits travaux sur matières végétales ou peaux, de fabrication d'objets en os ou pierre. Les éléments de faucille sont, quant à eux, absents du réduit et représentés à un exemplaire dans

51. En ce qui concerne les matières premières, $c f$. ASTRUC, 2002 ; ASTRUC et al., 2001.

52. Ces débris sont en partie le résultat de pollutions liées à l'architecture de terre.

53. ASTRUC, 2002 ; ASTRUC, sous presse a et b. 

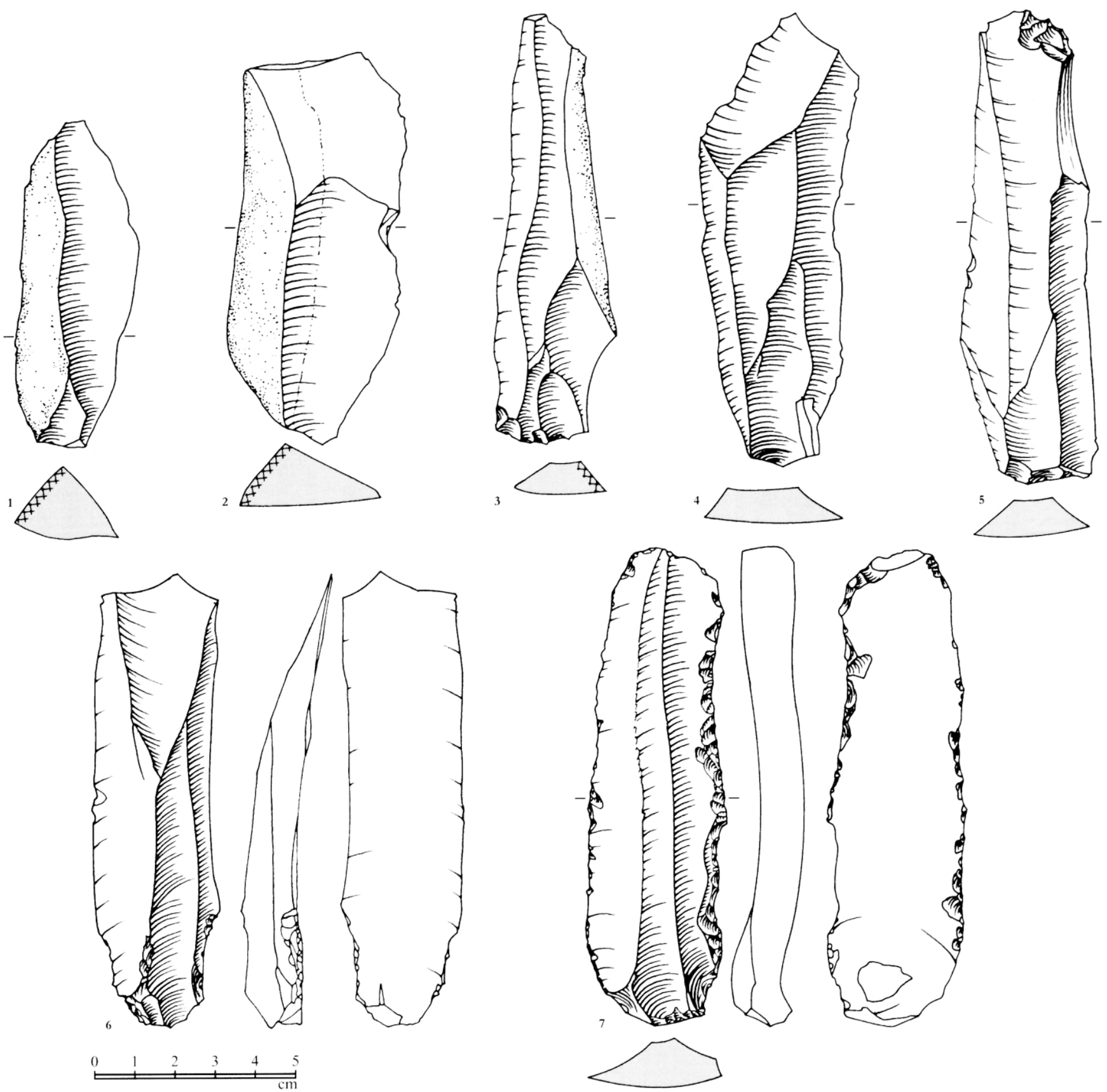

Fig. 8 : Khirokitia (Néolithique précéramique récent).

Construction S. 122, niveau C. Quelques exemples d'outils mis en réserve dans le réduit : 1-4, industrie laminaire sur plaquette de chert grenu, dominante sur le site : 5 -7. produits laminaires unipolaires et bipolaires, exceptionnels par leur qualité, en chert opaque.

l'habitation. Certaines catégories d'instruments identifiées dans le réduit sont en revanche absentes de tout autre contexte étudié. Trois lames de belle facture dont deux lames bipolaires (fig. 8: 5-7) y ont été recueillies. Réalisées sur une matière première locale, trouvée majoritairement sur rognons, elles sont issues de débitages représentant un niveau de savoir-faire et d'habileté bien supérieur à celui du reste de l'industrie; elles constituent ainsi un argument en faveur de 
l'hypothèse selon laquelle le choix d'une industrie lithique fondée sur des productions expédientes (en l'occurrence, laminaires) ne procède pas d'une absence des savoir-faire nécessaires à la conduite de débitages plus élaborés ${ }^{54}$. Des lames trapues associées au travail de la pierre et du bois ont, ensuite, été trouvées dans le réduit ; ces pièces correspondent à des travaux qui ne sont pas représentés dans le reste de l'assemblage étudié. Les lames trapues utilisées pour le travail de la pierre étaient dévolues à la décoration d'objets en pierre dure (vaisselles fines ou galets gravés); dans cette construction, des indices répétés témoignent de la fabrication de mobilier en pierre, un artisanat au sein duquel il semble que l'on puisse aujourd'hui démontrer l'existence de diverses échelles de production, d'opérations techniques à caractère plus ou moins spécialisé.

Un certain nombre de remarques s'imposent quant à la reconnaissance des contextes particuliers que sont les réserves. L'exemple que nous venons de décrire indique assez clairement que l'identification d'un comportement de mise en réserve n'est pas chose aisée. Ce comportement est visible grâce à un contexte architectural particulier et grâce à la conservation du dépôt au moins sur une bonne partie de son extension stratigraphique, peu ou pas perturbé par l'installation du sol suivant: impossible de l'identifier à un autre moment de l'occupation de cette construction pour laquelle, pourtant, l'agencement architectural reste similaire. On peut alors avancer que ce comportement devait être plus courant qu'il n'y paraît.

Il importe également de distinguer la réserve de S. 122 d'autres types de concentration de matériel observées à Khirokitia : deux concentrations d'outils particuliers interprétées comme des contextes de rejet. La première est constituée d'un petit ensemble de 14 éléments de faucille trouvé sur le radier d'une maison du niveau I, maison dont le sol n'a pas été conservé. Ces pièces déjà utilisées montrent une certaine homogénéité techno-morphologique (lames brutes, tronquées ou à dos) et pourraient provenir de trois ou quatre faucilles. La seconde concentration est composée de plusieurs dizaines d'outils lourds («bouchardes ») découverts sur les sols d'une maison du niveau B (S. 134) et d'un espace extérieur attenant ${ }^{55}$; l'ensemble présente, là encore, une forte homogénéité techno-typologique et fonctionnelle. Il se pourrait, en effet, qu'il s'agisse d'outils utilisés pour la fabrication de mobilier en pierre, notamment dans le cadre d'opérations techniques à caractère spécialisé (mise en forme et abrasion de vaisselles en pierre dure). Ce matériel n'est associé qu'à d'exceptionnels déchets techniques et ébauches de vaisselles.

\section{MODES DE CONSTITUTION ET RÔLES DES CONCENTRATIONS}

L'inventaire des données recueillies pour le Néolithique précéramique nous a conduits à proposer les définitions suivantes pour les termes de "dépôt», de "réserve" et de " cache » ${ }^{56}$. Le terme de " dépôt» fait référence à un ensemble clos, comportant un matériel homogène, sélectionné en vue d'une utilisation donnée. Celui de " réserve » a trait, en revanche, à un système ouvert : les biens emmagasinés sont souvent hétérogènes et sont utilisés au gré des besoins. L'emploi du terme « cache " apporte, quant à lui, une dimension toute particulière au comportement de stockage puisqu'il introduit les notions de dissimulation, de secret.

L'emploi de tel ou tel de ces termes constitue une interprétation qui repose sur un ensemble de critères. Ce choix terminologique est difficile; il est de plus loin d'être anodin: " dépôt » et « réserve » se placent, en effet, dans la sphère des pratiques utilitaires et économiques et renvoient aux comportements de stockage tandis que " cache » renvoie à des pratiques sociales largement influencées par les champs imaginaires et symboliques de chacune des communautés.

Les critères qui structurent les définitions proposées vont être discutés en abordant d'abord la question du mode de constitution de ces concentrations, puis celle de leur valeur utilitaire, économique, voire symbolique.

\section{MODES DE CONSTITUTION ET FONCTIONNEMENT}

Nous avons pu dégager deux types majeurs de comportement. 1. La sélection de pièces diverses (lames, éclats, nucléus, outils utilisés ou non) correspondant au tout venant de l'industrie recueillie par ailleurs sur le site ; 2 . la constitution d'ensembles homogènes (des supports bruts, le plus souvent, mais aussi plus exceptionnellement, des nucléus épuisés et pièces techniques ou encore, des pointes de flèche).

56. Pour chacun des termes, les traductions anglaises suivantes peuvent

être proposées : dépôt/deposit, réserve/reserve, cache/cache.
54. ASTRUC, sous presse a.

55. LE BRUN, sous presse. 
Dans le premier cas, il s'agit de réserves de matériel qui fonctionnent comme un système ouvert et dynamique, réduit ou alimenté au fur et à mesure des besoins: Khirokitia, l'exemple le mieux documenté, Gilgal 1 et Mureybet IIIA. Elles sont constituées d'éléments divers tous utilisables : des nucléus prêts à être débités et des outils neufs, peu utilisés ou déjà recyclés mais prêts à l'emploi. La diversité des ensembles indiquerait qu'ils sont constitués par les producteurs des outils et par leurs utilisateurs qui fréquentent le même lieu, la maison ou l'unité domestique. La réserve serait alors avant tout formée dans la maison pour des questions de commodité, afin que des outils produits à une certaine distance spatiale de la maison (espace extérieur, bord de rivière, affleurements) soient immédiatement disponibles. Tout indique que la maison ou l'unité domestique est relativement autonome dans la constitution de son outillage ; en d'autres termes, la distance sociale entre producteurs et utilisateurs est faible ${ }^{57}$.

Dans le second cas, le mode de constitution de la concentration est bien différent puisque des sélections très précises sont effectuées au sein des productions lithiques représentées sur le site; seuls les produits de certains segments de chaînes opératoires sont choisis. L'homogénéité interne des ensembles mis en réserve et les remontages plus fréquents indiquent une seule ou quelques séquences de formation. La disposition de lames en fagot va, en outre, dans le sens de cette interprétation et indique que les pièces ont pu être transportées ou sont prêtes pour le transport. Les situations rencontrées sont là assez diverses d'une concentration de lames à l'autre: des lames brutes (Jerf el Ahmar, 'Ain Ghazal, Halula, el-Kerkh), des lames brutes et retouchées (Abu Hureyra), des lames retouchées en pointes (Cheikh Hassan) et enfin, une situation plus diverse à Beidha, avec quelques lames à crête, des lames brutes ou légèrement retouchées, des pointes et deux nucléus-outils. L'exemple de Jerf el Ahmar qui présente plusieurs concentrations est particulièrement intéressant. Il montre en effet qu'une diversité importante peut exister au sein d'un même site, diversité qui s'exprime à la fois au niveau de l'origine technologique distincte des produits et au niveau du type d'objets sélectionnés: des lames communes (concentration 1), des nucléus à lame épuisés et pièces techniques associées (concentration 2), des lames prédéterminées (concentration 3 ).

Nous allons maintenant examiner les facteurs qui président à la constitution de ces concentrations en discutant du rôle utilitaire et du poids économique de ces ensembles.

57. ASTRUC, sous presse b et c.

\section{RÔLE UTILITAIRE ET POIDS ÉCONOMIQUE}

Le contexte précis d'utilisation, la destination fonctionnelle du matériel mis en réserve, est souvent difficile à saisir. Plusieurs constats s'imposent toutefois.

1. Les concentrations sont constituées dans l'aire domestique, formée d'une ou plusieurs constructions et des espaces extérieurs directement attenants.

2. Lorsque des analyses fonctionnelles ont été réalisées sur le matériel des concentrations, les utilisations s'inscrivent dans le fonctionnement de l'unité domestique (Mureybet IIIa) mais peuvent montrer quelques spécificités (Khirokitia).

3. Les études fonctionnelles conduites ont démontré que les outils correspondent à divers moments d'utilisation : ils sont, selon les cas, bruts, faiblement utilisés, ravivés, recyclés (mode de constitution 1, Mureybet IIIa et Khirokitia). Ces indications qui relèvent de la gestion de l'outillage et des cycles d'utilisation sont essentielles pour l'interprétation. L'exemple d'Halula (mode de constitution 2) illustre toutefois les difficultés devant lesquelles nous nous trouvons pour qualifier ces ensembles. Nous aurions en effet pu considérer le petit groupe de trois lames préférentielles comme un dépôt : des lames brutes sélectionnées en vue d'une utilisation future, un système clos. Mais, ces lames ont subi des cycles d'utilisation distincts (deux faiblement utilisées et une brute). Selon que la constitution de la concentration a eu lieu avant l'utilisation des lames ou après, nous nous trouvons devant un système clos ou ouvert.

4. Le matériel est, dans tous les cas, considéré comme toujours utilisable, à l'exception toutefois des nucléus épuisés de Jerf el Ahmar.

5. Aucun des exemples dont nous disposons ne semble correspondre à une situation parfois rencontrée en ethnologie : une réserve d'outils destinés à une activité très précise trouvée sur le lieu-même de cette activité58.

6. Le développement des analyses techno-fonctionnelles non seulement sur les concentrations mais aussi sur le reste de l'assemblage est nécessaire pour mieux caractériser ces ensembles. $\mathrm{Si}$, dans certains cas, la destination fonctionnelle des éléments lithiques peut être supposée en se fondant sur des critères technologiques (lames de Tell el-Kerkh destinées à la moisson) ou critères typologiques (pointes de Cheikh Hassan destinées à la chasse), de telles hypothèses méritent d'être vérifiées.

58. Cf. par exemple, dans le cas de l'artisanat de la peau au Maroc, IBÁÑEZ et al., 2002. 
Ainsi, contrairement à ce que l'on aurait pu attendre, les concentrations de matériel ne présentent en général rien d'exceptionnel : les matières premières employées, les connaissances et savoir-faire mis en jeu lors de la production ou encore l'utilisation des produits lorsque celle-ci peut-être identifiée sont équivalentes à ce qui a été observé par ailleurs sur chacun des sites. Les facteurs technologiques ne semblent donc pas être les plus déterminants lors de la constitution des concentrations : ces dernières ne sont pas formées en priorité pour prévenir un risque ${ }^{59}$ lié à une accessibilité limitée au matériel - difficulté d'accès aux matières premières ou savoir-faire et connaissances requis pour la production peu partagés. Les ensembles de Jerf el Ahmar indiquent la même tendance puisque sont mis de côté non seulement des lames communes, mais aussi des lames prédéterminées, produits laminaires plus rares correspondant à un type de débitage nouveau qui n'est peut-être maîtrisé que par quelques-uns.

Si le rôle utilitaire de ces ensembles est indéniable, ils ne présentent en revanche pas, à notre avis, de caractère symbolique marqué. Les lames prédéterminées découvertes dans une niche scellée - seul contexte pour lequel le terme de " cache " tel que nous l'avons défini puisse éventuellement être utilisé ou encore les éléments lithiques découverts dans les enduits à Jerf el Ahmar constituent des indices pouvant être pris en compte dans une discussion sur le rôle symbolique du lithique taillé pour ces périodes. Ils restent relativement faibles lorsque l'on se réfère à d'autres pratiques bien attestées : les bucranes placés à l'intérieur de banquettes de bâtiments collectifs sur le même site, ceux qui étaient placés sous des piliers ou des habitations à Mureybet ${ }^{60}$, ou encore les dépôts de bucranes et de crânes humains retrouvés à Tell Abr $3^{61}$.

Enfin, tous les éléments dont nous disposons à l'heure actuelle indiquent que l'impact économique des concentrations est faible; le matériel réuni représente un volume de production peu important ${ }^{62}$ et correspond le plus souvent à des activités de taille assez largement représentées dans l'habitat. Il reste en outre probablement à usage individuel ou domestique, à l'exception peut-être de quelques concentrations qui peuvent être destinées à être redistribuées dans un cadre plus large (Abu Hureyra ou Bouqras).

Nous considérons que la constitution des concentrations reflète ces mécanismes de production et de redistribution et

59. TORRENCE, 1989

60. Cauvin, $1994: 120$.

61. T. Yartha., comm. pers.

62. Les exemples de concentration dont nous disposons comprennent de trois à trois cent éléments. qu'elle est largement influencée par ce que nous appelons la distance entre producteur et utilisateur. Cette distance peut être d'ordre géographique (région), spatiale (intrasite), mais elle est aussi, et plus certainement, sociale.

\section{REFLET DES MÉCANISMES DE PRODUCTION ET DE REDISTRIBUTION DE L'OUTILLAGE}

La question fondamentale qui se pose lorsque l'on traite du matériel lithique taillé est la suivante : comment un individu, une cellule domestique acquièrent-ils leur outillage? en d'autres termes, quels sont au Néolithique précéramique au Proche-Orient les mécanismes de production et de redistribution de l'outillage ? Nous verrons que ces mécanismes évoluent tout au long de la séquence étudiée, du PPNA à la fin du PPNB.

Nous considérons d'abord que les deux modes de constitution des concentrations ne sont pas exclusifs. Le premier type de comportement, la mise en place de réserves, est observé dans des contextes chronologiques et culturels divers puisqu'on le retrouve aussi bien sur l'Euphrate et dans la vallée du Jourdain au PPNA qu'à Chypre au Néolithique précéramique récent ; à chaque fois, le matériel des réserves est le reflet de l'assemblage de chacun des habitats et aucune sélection particulière ne semble dominer ( $c f$. supra). Ces réserves sont constituées à la fois par les producteurs et les utilisateurs, dans une logique de production et d'utilisation. Que se passet-il quand les éléments mis de côté sont plus soigneusement sélectionnés, comportement qui est présent dès la période de transition entre PPNA et PPNB et qui perdure?

Deux ruptures importantes sont observées par rapport au PPNA, période à laquelle le matériel stocké correspondait au tout venant de la production :

- Les produits laminaires sélectionnés sont de bonne qualité et issus le plus souvent de débitages bipolaires (Jerf el Ahmar, Cheikh Hassan, Beidha, Halula, 'Ain Ghazal), plus rarement unipolaires (Cheikh Hassan, Tell el-Kerkh ${ }^{63}$ ) ou encore de débitages par pression dans le cas de l'obsidienne (Sabi Abyad II et Bouqras); les nucléus stockés sont, quant à eux, bipolaires (Jerf el Ahmar, Akarçay Tepe).

- Des produits particuliers issus de segments très précis des chaînes opératoires sont sélectionnés ce qui détermine pour chacun des exemples connus des contenus homogènes,

63. La technique de taille serait, dans le cas de Tell el-Kerkh, soit la pression, soit la percussion indirecte (ARIMURA, sous presse). 
même si les situations rencontrées restent pourtant très diverses d'une concentration à l'autre (cf. supra).

La première rupture correspond directement à une évolution majeure des systèmes de production de l'outillage; la phase de transition entre PPNA et PPNB voit en effet l'adoption de nouvelles méthodes de débitage laminaire. Les nucléus bipolaires ou unipolaires sont conçus pour produire un nombre plus limité de lames plus fortement standardisées et qui partagent deux caractères, un profil parfaitement rectiligne et une extrémité distale aiguë ${ }^{64}$. Aux alentours de 8700 $8200 \mathrm{cal}$. BC, la production de lames prédéterminées issues de débitages naviformes est une pratique qui devient dominante dans tout le Levant. Cette taille bipolaire plus élaborée a toujours coexisté avec des débitages plus simples ${ }^{65}$.

La seconde rupture indique qu'il existe alors une césure entre la production et l'utilisation des lames. À partir de la transition entre PPNA et PPNB, les stocks sont constitués avant tout avec une logique d'utilisateur. Les contextes mixtes destinés à la production et à l'utilisation sont absents. Seuls les deux ensembles de nucléus à Jerf el Ahmar et à Akarçay pourraient être considérés comme des stocks constitués par des producteurs. Mais l'on attendrait plutôt des concentrations où nucléus et outils utilisés pour la taille (percuteurs, abraseurs) seraient associés, et qui se trouveraient à proximité de structures ou de zones spécifiquement dévolues à la taille - des ateliers au sens strict ou des accumulations significatives de déchets techniques (nucléus épuisés, tablettes, etc.). La césure observée serait d'ordre temporel et spatial comme dans le cas du premier type de comportement mis en évidence, mais elle serait ici surtout d'ordre social : les connaissances et savoir-faire nécessaires à la production laminaire de qualité ne sont pas l'apanage de tous, mais plutôt le fait d'une partie du groupe. À quel type d'organisation sociale de la production et de la redistribution de l'outillage est-il alors possible de faire référence?

Les données dont nous disposons sur ces contextes interprétés comme de possibles dépôts, réserves ou caches indiquent que nous nous trouvons, semble-t-il, devant un système de production lithique de type domestique ${ }^{66}$. Ce système est défini de façon suffisamment générale pour que les différents cas de figure observés au cours du Néolithique précéramique puissent y être rattachés : les tailleurs sont des artisans qui travaillent à temps partiel au sein de la communauté. Nous rejoignons en cela la définition récemment proposée pour la

64. ABBĖs, $2003: 54,67$.

65. Cette dualité a notamment été évoquée pour 'Ain Ghazal (QUINTERO, 1998).

66. PEACOCK, 1982 : 8; VAN DER LeUW, 1984 ; RICE, 1987 : 184. spécialisation par C. Perlès : " Specialization is here defined as an activity done by a limited number of groups or individuals, in order to redistribute the products (or services) within a wider community. It usually, but not necessarily, rests upon knowledge, skills or equipment not possessed by the others. Craft specialization, thus defined, can go hand by hand with other subsistence activities and need not be full time $»^{67}$.

Dans ces sociétés précéramiques, la production de l'outillage en silex reste cantonnée à une petite échelle (cf. supra, rôle économique). Dans la majorité des cas, une diversité des situations au sein même des communautés est supposée : des degrés divers de spécialisation ou différentes échelles de production devaient être présents ${ }^{68}$; l'exemple des concentrations de Jerf el Ahmar constitue une belle illustration de cette diversité intracommunautaire, les ponctions au sein des chaînes opératoires pour la constitution des concentrations sont de nature très variables. Mais, le degré de spécialisation reste faible même dans le cas des productions laminaires bipolaires: une part non négligeable du groupe serait capable de conduire ces débitages complexes et chacun des artisans échangerait ses productions avec des membres du groupe extérieurs à sa propre unité domestique. Cette distribution des lames ne dépasse sans doute pas l'échelle de petits services ou cadeaux entre "voisins ", qui sont le propre des degrés les plus faibles de spécialisation ${ }^{69}$.

Il s'agit probablement d'une spécialisation naissante ${ }^{70}$, dont nous avons des témoins dès la transition entre le PPNA et le PPNB et qui semble s'intensifier à partir du PPNB moyen et surtout au PPNB récent/final. C'est d'ailleurs à ce moment qu'apparaissent les dépôts comportant un nombre plus important de lames ('Ain Ghazal, Abu Hureyra, Bouqras et Tell elKerkh), souvent disposées en fagot ce qui facilite le transport. Ces concentrations fonctionnent comme des systèmes clos ; elles comprennent une quantité de supports qui paraît supérieure aux seuls besoins de l'unité domestique ce qui induit l'existence d'échanges au sein de la communauté.

Les concentrations de matériel lithique reflètent aussi l'existence d'échanges extra-communautaires. La distance entre producteur et utilisateur est alors importante et l'on se situe là à l'opposé du mécanisme de production et de redistribution enregistré à l'échelle domestique dans des cas comme ceux de Khirokitia, de Gilgal 1 ou de Mureybet IIIA.

\footnotetext{
67. PERLĖs, $2001: 208$, note 19

68. Cf. Khirokitia, supra.

69. GONZÁLEZ et al., 2001

70. "Incipient specialization », $c f$. NISHIAKI, 2000
} 
Les matériaux qui circulent entre différentes communautés témoignent eux-mêmes de réseaux de plus ou moins grande échelle. La diffusion de l'obsidienne constitue certainement l'un des exemples les plus complexes ${ }^{71}$. Le matériau circule sur de longues distances sous forme de produits finis, mais peut aussi faire l'objet de productions locales comme cela a été mis en évidence à Mureybet, Cheikh Hassan, Qdeir, el Kowm 2, Aswad et à Akarçay Tepe ${ }^{72}$. À Sabi Abyad II et à Bouqras, l'obsidienne est introduite sous forme de produits finis et les exemples de stock identifiés sur chacun de ces sites indiqueraient deux échelles distinctes d'acquisition et de redistribution de ce mobilier au sein de la communauté. À Sabi Abyad II, nous nous trouverions devant un stock individuel destiné à l'unité domestique. À Bouqras, les trois cents supports représentent un volume important à l'échelle d'un site receveur. Ils n'étaient vraisemblablement pas destinés à la seule consommation de la maison où ils ont été trouvés mais à être redistribués au sein de la communauté.

Contrairement à ce que l'on aurait pu attendre ces matériaux exogènes ne semblent pas stockés ou mis en réserve en priorité : nous ne disposons que de deux exemples de concentrations d'obsidienne, de quelques indices pour les lames en silex chocolat ${ }^{73}$ et d'aucune mention pour le silex rosé ou mauve ${ }^{74}$.

\section{CONCLUSION}

Les concentrations lithiques mentionnées sont encore rares ; elles se répartissent du PPNA au PPNB final sur le continent (14), et au Néolithique précéramique récent à Chypre (1). Il s'agit de cas extrêmement divers par leur localisation, leur contenant et leur contenu. Les concentrations sont, de plus, décrites de façon plus ou moins détaillée, de la simple mention, situation la plus fréquente, à une analyse technologique et fonctionnelle complète. Ce manque de données rend l'interprétation

71. BALKAN-ATLi et al., 1998; BINDER and BALKAN-ATLI, 2001 ; BRIOIS et al., 1997.

72. CAUVIN, 1998: 265; ABBĖS et al., 2001 ; ABBĖS, sous presse ; STORDEUR et al., sous presse.

73. Le silex chocolat faisait vraisemblablement l'objet d'échanges extracommunautaires: l'absence de témoins de débitage sur des sites où des produits finis, et notamment des pointes, sont abondants a été signalée (par exemple, sur le Moyen Euphrate, au PPNB, à Tell Halula et Akarçay tepe).

74. La situation est inverse pour les silex roses ou mauves dont les sources seraient situées à plus de $200 \mathrm{~km}$ de Mureybet et Cheikh Hassan ; la production est réalisée sur les sites comme l'attestent fragments de nucléus, éclats corticaux, esquilles et lames techniques. difficile au cas par cas; le choix des termes - « dépôts », " caches ", "réserves »- est pour cela le plus souvent périlleux, les critères qui fondent ces définitions n'étant pas toujours caractérisés, en particulier, les aspects qui relèvent de la destination fonctionnelle des outils stockés - opérations techniques et cycles d'utilisation. Seules des réserves ont pu être clairement identifiées à Gilgal 1, Mureybet IIIA et Khirokitia ; les autres cas sont plus difficiles à interpréter ( $c f$. supra).

L'analyse que nous avons conduite a, en revanche, permis de démontrer que l'étude techno-fonctionnelle de tels ensembles était hautement souhaitable pour analyser les mécanismes de production et de redistribution de l'outillage au sein des communautés du Néolithique précéramique. Nous avons pu montrer, en effet, que parmi les facteurs - technologiques, économiques, sociaux - qui président à la constitution des concentrations, les derniers dominaient.

Contrairement à ce que l'on aurait pu attendre, le matériel stocké est réuni dans des aires domestiques, le plus souvent dans une logique d'utilisation. Il est commun et reflète généralement le reste de l'assemblage représenté sur le site. Ces stocks peu importants numériquement ont un rôle utilitaire certain. Ils constituent sans nul doute un moyen de gestion de l'outillage mais dans des situations où il n'existe pas nécessairement de risques pesant sur la fabrication de l'outillage : une acquisition des matières premières difficiles ou des connaissances ou savoir-faire nécessaires à la production peu partagés. Le type de sélection opéré au sein des chaînes opératoires représentées sur le site nous a permis de mettre en évidence deux modes de constitution majeurs pour ces concentrations.

Quel que soit le contexte étudié, nous nous trouvons semble-t-il devant un système de production de type domestique. Toutefois, à partir de la transition PPNA/PPNB, le comportement de stockage évolue avec une sélection précise d'éléments lithiques au sein des chaînes opératoires ce qui détermine des ensembles caractérisés par une forte homogénéité interne. Les produits laminaires sont alors plus fréquents; cette évolution correspond à celle des débitages à cette période. Elle correspond également à l'apparition de différentes échelles de production ou différents degrés de spécialisation au sein des activités de taille. Ces productions seraient échangées par les producteurs avec des consommateurs qui n'appartiennent plus seulement à l'unité domestique mais à un cercle élargi de «voisins». Les quelques stocks de supports en obsidienne reconnus témoigneraient, quant à eux, d'une volonté de gestion de l'outillage dans le cadre d'échanges qui peuvent dépasser le cadre communautaire, cette fois. Ainsi, l'utilisateur pour acquérir son outillage serait devant une gamme de possibilités 
plus ou moins large selon l'organisation de la communauté à laquelle il appartient, de l'échelle individuelle ou domestique aux échanges intra et extra-communautaires.

Toutes les concentrations mentionnées ont enfin une portée économique très faible ce qui va à l'encontre de l'hypothèse de l'existence d'une spécialisation économique au $\mathrm{PPNB}^{75}$. Les concentrations de matériel lithique que nous avons mentionnées s'intègrent en revanche mieux, à notre avis, dans le cadre d'une hypothèse fondée sur l'existence, d'une part, d'un système de production domestique et d'autre part, d'un système d'échange organisé à différentes échelles.

\section{REMERCIEMENTS}

Cet article n'aurait pu voir le jour sans Danielle Stordeur, MarieClaire Cauvin, P.M.M.G. Akkermans, Alain Le Brun, Miguel Molist et Marc Verhoeven qui nous ont confié l'étude du matériel de leurs sites. Les illustrations ont été réalisées avec le concours de Chantal Perrot (fig. 1), d'Odile Le Brun (fig. 7) et de Jacqueline Courbet (fig. 8). Les dessins sont de Gérard Deraprahamian (fig. 2-5) et de Gérard Monthel (fig. 6). Nous remercions également Gérard Monthel qui a repris l'ensemble des figures pour les mettre en page.

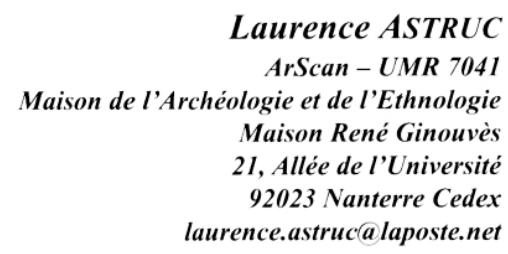

Frédéric ABBÈS

Archéorient - UMR 5647

Maison de l'Orient et de la Méditerranée

7 , rue Raulin

69007 Lyon

abbes@mom.fr

Juan Jose IBÁÑEZ ESTÉVEZ, Jesús GONZÁLEZ URQUIJO

Departamento de Ciencias Históricas Grupo de Prehistoria. Universitad de Cantabria Avda. de los Castros, s.n. 39005 Santander ibanezij@unican.es gonzalje@unican.es

\section{BIBLIOGRAPHIE}

ABBÈS F.

2003 Les outillages néolithiques en Syrie du Nord: méthode de débitage et gestion laminaire durant le PPNB. Oxford Lyon: BAR International/Maison de l'Orient 1150. sous presse Tell Qdeir 1: un exemple de débitage d'obsidienne dans la steppe Syrienne. In : BALKAN-ATLI N. and BINDER D. (eds), Neolithic Chipped Stone Industries of the Fertile Crescent, Proceedings of the $4^{\text {th }}$ Workshop on PPN Lithic Industries, Nigde, 4-8 June 2001. SENEPSE. Berlin : ex oriente.

AbBĖs F., Cauvin M.-C., Gratuze B., Bellot-Gurlet L., BRESSY C. et POUPEAU G.

2001 Nouvelles recherches sur l'obsidienne de Cheikh Hassan (Vallée de l'Euphrate, Syrie) au Néolithique: PPNA et PPNB ancien. Syria $78: 5-17$.

\section{ARIMURA M.}

2003 Chipped stone artifacts. In : IWASAKI T. and TSUNEKI A. (eds), Archaeology of the Rouj Basin, a regional study of the transition from village to city in the northwest Syria. Study for West Asian Archaeology 1 : 57-97. Al-Shark 2, University of Tsukuba.

sous presse LPPNB blade cache at Neolithic Tell Ain el-Kerkh, Northwest Syria. In : BALKAN-ATLi N. and BINDER D. (eds), Neolithic Chipped Stone Industries of the Fertile Crescent, Proceedings of the $4^{\text {th }}$ Workshop on PPN Lithic Industries, Nigde, 4-8 June 2001. SENEPSE. Berlin : ex oriente.

Arimura M., Balkan-Atli N., Borrell F., Cruells W., Duru G., ERIM-ÖZdOGAN A., IBȦÑEZ J.J., MAEDE O., MIYAKE Y.,

MOLIST M. and ÖZBASARAN M.

2000 A new Neolithic settlement in the Urfa region: Akarçay Tepe, 1999. Anatolia Antiqua VII : 227-255.

ASTRUC L.

2002 Analyse fonctionnelle et spatiale de l'outillage taillé de Khirokitia, Néolithique précéramique récent de Chypre. Monographies du CRA 25. Paris: CNRS-Éditions.

sous presse a L'outillage lithique taillé de Khirokitia (Néolithique précéramique récent, $\mathrm{VII}^{\mathrm{e}}$ millénaire av. JC calibré) : caractéristiques générales et particularités. In: GUILAINE J. et LE BRUN A. (éd.), Le Néolithique de Chypre. Colloque international, Nicosie, 17-19 mai 2001. Athènes: École française d'Athènes.

sous presse b Des pointes de flèche aux faucilles. Mutations économiques et sociales au VII millénaire av. JC en Méditerranée orientale : l'exemple de la vallée du Balikh (Syrie du Nord). Annales de la fondation Fyssen 18

sous presse c Points and glossed pieces from tell Sabi Abyad II and tell Damishliyya I, (Balikh valley, Northern Syria). In : BALKAN-ATLI N. and BINDER D. (eds), Neolithic Chipped Stone Industries of the Fertile Crescent, Proceedings of the $4^{\text {th }}$ Workshop on PPN Lithic Industries. Nigde, 4-8 June 2001. SENEPSE. Berlin : ex oriente.

ASTRUC L., JAUtÉE E., VARgiolu R. et Zahouani H.

2001 La texture des matières siliceuses et son influence sur la nature et le développement des traces d'usure : apports des méthodes expérimentales. L'exemple des cherts de la formation de Lefkara (Chypre). In : BOURGUIGNON V., ORTEGA I. et FrĖre-SAUtOT M.-Ch. (éd.), Préhistoire et approche expérimentale: 233-257. Montagnac: Éditions Monique Mergoil.

AURENCHE O.

1977 Un exemple de l'architecture domestique en Syrie au VIII millénaire : la maison XLVII de Mureybet (Syrie). In : MARGUERON J.-C. (éd.), Le Moyen Euphrate, zone de contacts et d'échanges. Travaux du centre de recherche sur le Proche-Orient et la Grèce antique 5: 35-53. Leiden : Brill.

\footnotetext{
75. QUINTERO and WILKE, 1995 ; QUINTERO, 1998.
} 
BALKAN-ATli N., Binder D. et KuZuCUOGLU C.

1998 Exploitation de l'obsidienne de Cappadoce : Première campagne de fouille à Kaletepe (Kömürcü). Anatolia Antiqua VI : 301-315.

BANNING E.B. and BYRD B.F.

1987 Houses and the changing residential unit : domestic architecture at PPNB 'Ain Ghazal, Jordan. Proceedings of the Prehistoric Society $53: 309-325$.

BAR-YOSEF O. and ALON D.

1988 Nahal Hemar cave. 'Atiqot. English series XVIII. Jerusalem.

BINDER D. and BALKAN-ATLI N.

2001 Obsidian and blade technology at Kömürcü-Kaletepe (Cappadocia, Turkey). In : CANEVA I., LEMORINI C., ZAMPETTI D. and BIAGGI P. (eds), Beyond tools : redefining the PPN lithic assemblages of the Levant. SENEPSE 9: 1-16. Berlin : ex oriente.

Briois F., Gratuze B. et Guilaine J.

1997 Obsidiennes du site néolithique précéramique de Shillourokambos (Chypre). Paléorient 23,1 : 95-112.

CAUVIN J.

1994 Naissance des divinités, Naissance de l'agriculture. La Révolution des Symboles au Néolithique. Paris : CNRS-Éditions, Collection « Empreintes ».

\section{CAUVIN M-C.}

1998 L'obsidienne : données récentes provenant de sites-habitats néolithiques. In: CAUVIN M.-C., GOURGAUd A., Gratuze B., Arnaud N., Poupeau G., POIDEVIN J.-L. et Chataigner C. (éd.), L'obsidienne au Proche et Moyen Orient: du volcan à l'outil. BAR Int. Ser. 738:259-271.

CAUVIN M.C., ABBĖS F., IBȦÑEZ J.J. et GonZȦLEZ J.E.

2001 L'outillage lithique dans la maison du niveau IIIa, PPNA, de Tell Mureybet. In : CANEVA I., LEMORINI C., ZAMPETTI D. and BIAGGI P. (eds), Beyond tools : redefining the PPN lithic assemblages of the Levant. SENEPSE $9: 217-242$. Berlin : ex oriente.

\section{CONOLLY J}

1999 The Catalhöyük Flint and Obsidian Industry: Technology and Typology in Context. BAR Int. Ser. 787. Oxford.

2003 Catalhöyük Hoards : A contextual analysis of Technology. In : MONOLEY N. and SHOTT M. (eds), Lithic analysis at the Millennium : 55-78. London : Institute of Archaeology, University College.

COPELAND L.

2000 The Flint and Obsidian Industries. In : VERHOEVEN M. and AKKermans P.M.M.G. (eds), Tell Sabi Abyad II. The PrePottery Neolithic B Settlement. Report on the Excavations of the National Museum of Antiquities Leiden in the Balikh Vallev, Syria: 51-81. Istanbul : Nederlands Historish-Archaeologisch Instituut.

Coqueugniot E.

2000 Dja'de (Syrie), un village à la veille de la domestication (seconde moitié du 9 e millénaire av. JC.). In : GUILAINE J. (éd.), Les premiers paysans du monde, naissance des agricultures: 63-79. Paris : Errance.

DIKAIOS P

1953 Khirokitia. Final Report on the Excavation of a Neolithic Settlement in Cyprus on behalf of the Department of Antiquities 1936-1946. Monographs of the Department of Antiqui- ties of the Government of Cyprus 1. London, Oxford: University Press.

GonzÁlez UrquiJo J.E., IBÁÑez J.J., ZAPATA L. y PEÑA ChOCARRo L. 2001 Estudio etnoarqueológico sobre la cerámica Gzaua (Marruecos). Técnica y contexto social de un artesanado arcaico. Trabajos de Prehistoria 58,1: 5-31.

GORING-MORRIS N. and BELFER-COHEN A.

2001 The symbolic realms of utilitarian material culture : the role of lithics. In: CANEVA I., LEMORINI C., ZAMPETTI D. and BIAGGI P. (eds), Beyond tools : redefining the PPN lithic assemblages of the Levant. SENEPSE $9: 257-271$. Berlin : ex oriente.

IBÁÑEZ J.J., GONZÁLEZ URQUIJO J.E. et MORENO M

2002 Le travail de la peau en milieu rural : le cas de la Jebala Marocaine. In : AUdOUIN-ROUZEAU F. et BEYRIES S. (éd.), Le travail du cuir de la Préhistoire à nos jours. XXII rencontres internationales d'archéologie et d'histoire d'Antibes : 79-97. Antibes : Éditions APDCA.

IBÁÑEZ J.J., BORRELl F., MOLIST M. and BALKAN-ATLI N.

sous presse Lithic tools in Akarçay Tepe (Turkey). Technical evolution between 9000 and $7000 \mathrm{BP}$ in the mid Euphrates valley. In : BALKAN-ATLI N. and BINDER D. (eds), Neolithic Chipped Stone Industries of the Fertile Crescent, Proceedings of the $4^{\text {th }}$ Workshop on PPN Lithic Industries, Nigde, 4-8 June 2001. SENEPSE. Berlin : ex oriente.

KIRKBRIDE D

1967 Beidha 1965. An Interim report. Palestine Exploration Quarterly $99: 5-13$

LE BRUN A

1984 Fouilles récentes à Khirokitia (Chypre), 1977-1981. Paris : Éditions Recherche sur les Civilisations, mémoire n ${ }^{\circ} 41$.

1989 Fouilles récentes à Khirokitia (Chypre), 1983-1986. Paris : Éditions Recherche sur les Civilisations, mémoire n ${ }^{\circ} 81$.

1994 Fouilles récentes à Khirokitia (Chypre), 1988-1991. Paris : Éditions Recherche sur les Civilisations, Études néolithiques.

sous presse Khirokitia. Bulletin de Correspondance Hellénique 126.

LEVY J.

1982 Social and religious Organization in Bronze Age Denmark. An Analysis of Ritual Hoard finds. BAR Int. Ser. 124. Oxford.

Molist M., Ferrer A., GonzÁlez J.E., IBÁÑEZ J.J. et PAlOMO A

2001 Élaboration et usage de l'industrie lithique taillée de Tell Halula (Syrie du nord) depuis 8700 jusqu'à $7500 \mathrm{BP}$ : état de la recherche. In : CANEVA I., LEMORINI C., ZAMPETTI D. and BIAGGI P. (eds), Beyond tools: redefining the PPN lithic assemblages of the Levant. SENEPSE 9 : 243-256. Berlin : ex oriente.

MOORE A.M.T.

1975 The excavation of Tell Abu Hureyra in Syria : a preliminary report. Proceedings of the Prehistoric Society 41 : 50-77.

MOORE A.M.T., Hillman G.C. and LEGGE A.J.

$2000 \quad$ Village on the Euphrates. Oxford : Oxford University Press.

MORTENSEN P.

1988 A note on a small box with flint blades and arrowheads from Beidha and its implications. In: GARRARD A.N. and GEBEL H.G. (eds), The Prehistory of Jordan. The state of research in 1986. BAR Int. Ser. 396(i) : 199-207. Oxford.

NISHIAKI Y.

2000 Lithic Technology of Neolithic. BAR Int. Ser. 840. Oxford. 
NOY T. and KOZLOWSKI S.K.

1996 A Basket of Flint Artefacts from House 11 at Gilgal I, Locus 37/42. In : KOZLOWSLI S.K. and GeBEL H.G.K. (eds), Neolithic Lithic Stone Industries of the Fertile Crescent and Their Contemporaries in Adjacent Regions. SENEPSE 3 : 271-288. Berlin : ex oriente.

PEACOCK D.P.S

1982 Pottery of the Roman world: An ethnoarchaological approach. Londres : Longmans.

PELTENBURG E.

1991 A ceremonial Area at Kissonerga. Lemba Archaeological Project vol. II.2. Studies in Mediterranean Archaeology LXX : 3. Göteborg : Paul Åströms Förlag.

PERLES C.

2001 The Early Neolithic in Greece. Cambridge : Cambridge University Press.

QUINTERO L.

1998 Evolution of Lithic Economies in the Levantine Neolithic: Development and Demise of Naviform Core Technology. University of California, Riverside : Unpublished doctoral dissertation.

QUINTERO L.A. and WILKE P.J.

1995 Evolution and Economic Significance of Naviform Core and Blade Technology in the Southern Levant. Paléorient 21,1: 17-34.

RICE P.M.

1987 Pottery analysis. A Sourcebook. Chicago : The University of Chicago Press.

ROODENBERG J.J.

1986 Le Mobilier en pierre de Bouqras. Utilisation de la pierre dans un site néolithique sur le Moyen Euphrate (Syrie). Lei- den, Nino: Nederlands Instituut voor het Nabije Oosten/ Netherlands Institute for the Near East.

SIMMONS A.H. and NAJJAR M

1999 Preliminary field report of the 1998-1999 Excavations at Ghwair I, a Pre-Pottery Neolithic B community in the Wadi Feinan region of southern Jordan. Neo-Lithics 1/99 : 4-6.

2000 Preliminary Report of the 1999-2000 Excavation Season at the Pre-Pottery Neolithic Settlement of Ghwair I, Southern Jordan. Neo-Lithics 1/00:6-8.

STORDEUR D. et ABBĖS F.

2002 Du PPNA au PPNB : mise en lumière d'une phase de transition à Jerf el Ahmar, Syrie. Bulletin de la Société Préhistorique de France 99,3 : 563-595.

Stordeur D., ABbès F., Helmer D., Jammous B. et Willcox G.

A paraître Reprise des fouilles à Tell Aswad de Damascène : résultats préliminaires. In : MARGUERON J.C., MIROSCHEDJI P. de et Thalmann J.P. (éd.), Actes du $3^{e}$ Congrès international d'Archéologie du Proche-Orient ancien (3ICAANE), Paris 15-19 avril 2002. Winona Lake IN : Eisenbrauns.

TORRENCE R.

1989 Time, Energy and Stone Tools. Cambridge : Cambridge University Press.

\section{VAN DER LEEUW S.E.}

1984 Pottery manufacture : some implications for the study of trade. In : RICE P.M. (ed.), Pots and potters: current approaches in ceramic archaeology : 55-69. Los Angeles : University of California Press.

VERHOEVEN M. and AKKERMANS P.M.M.G.

2000 Tell Sabi Abyad II. The Pre-Pottery Neolithic B Settlement. Report on the Excavations of the National Museum of Antiquities Leiden in the Balikh Valley, Syria. Istanbul : Nederlands Historish-Archaeologisch Instituut. 
